(ID: S8-4)

\title{
Otitis media observed in unexpected natural death of infants
}

Yoshiaki Hashimoto, Junichi Furumiya

Department of Legal Medicine, Kochi Medical School, Kochi University,

Kohasu, Oko-cho, Nankoku City,

Kochi 783-8505,

Japan

Corresponding author: Junichi Furumiya

Department of Legal Medicine, Kochi Medical School, Kochi University,

Kohasu, Oko-cho, Nankoku City,

Kochi 783-8505,

Japan

Tel: +81 88880 2419; fax: +81 888802420

E-mail address: furumiya@kochi-u.ac.jp 


\section{Abstract}

Intracranial complications due to otitis media such as brain abscess and leptomeningitis are well known as a cause of death. In recent years, encountering those serious intracranial complications in forensic medical practice is extremely rare. However, we rarely encounter autopsy cases with otitis media of which pathological damage is mild or moderate. We investigated 11 autopsy cases ( 6 cases of pneumonia and 5 cases of SIDS) in unexpected natural death of infants under one year old, and found 3 cases with otitis media. The tympanic cavity was investigated by chiseling a petrosal part of the base of the skull. In the case of otitis media, serous and mucous exudate containing leucocytes examined microscopically was observed. Otitis media, as such, was not a cause of death in our cases presented. Background factors causing otitis media seems to be not only functional insufficiency of the auditory tube but also other delicate constitution, hidden dysfunction or immature function in immune system, which could be easily infected. Of three cases of otitis media, cytomegalovirus infection was observed in two cases simultaneously. In our department, we have little opportunity to encounter autopsy cases of infant under one year old. If many infant cases could be investigated, many autopsy cases with otitis media might be encountered in unexpected infant deaths.

Keywords: Forensic pathology; Otitis media; Cytomegalovirus; Pneumonia; SIDS 


\section{Materials and Methods}

2.1. Human autopsy cases

\subsubsection{Investigated subjects}

We investigated 26 autopsy cases of infants under one year old. Of those, unexpected 
natural death were 11 cases (SIDS in 5 cases, pneumonia in 6 cases) and unnatural death due to traumatic causes or neglect were 15 cases (homicide in 14 cases, accidental death in 1 case). For determining otitis media and cytomegalovirus infection, the tympanic cavity and the parotid grand were investigated in 11 cases of unexpected natural death.

In the cases with otitis media, we examined family information about age, sex, period of death, cause of death, parents age, passive smoking, number of siblings, birth weight, gestational age, deliver type, breast-feeding.

\subsubsection{Procedure to investigation}

The tympanic cavity was investigated by intracranial approach, and was opened by chiseling superficial bone fragment in bilateral petrosal part of the base of the skull to the degree of exteriorizing auditory ossicle. Under normal condition, the tympanic cavity is emptiness. When fluid was observed in the tympanic cavity, it was collected with a syringe or cotton application depending on the fluid volume, and a smear of the fluid was examined under a microscope following hematoxylin-eosin staining.

To investigate into cytomegalovirus infection in the parotid grand and pathological conditions in other organs, the tissues were observed microscopically following Hematoxylin-eosin staining.

\subsection{Toxicological screening}

Blood was processed for drugs screening by gas chromatography. 


\section{Result}

Table 1 shows age, sex, cause of death, with or without of otitis media and

cytomegalovirus infection in the parotid grand in 11 infants under one year of age with unexpected natural death. The average of postnatal age in 11 cases was 4.6 months of age, and it was in the same average in pneumonia or SIDS. Causes of death were pneumonia in 6 cases and SIDS in 5 cases. Of 11 cases, otitis media was observed in 3 cases (27\%), and it occurred in bilateral tympanic cavity in all of 3 cases. The fluid observed in the tympanic cavity was viscid serous exudate in 2 cases (case no.2 and 11 in Table 1) and viscid purulent fluid with palish yellow in color in 1 case (case no. 7 in Table 1).

Of 11 cases, cytomegalovirus in the parotid grand was observed in 3 cases (27\%). Otitis media accompanying cytomegalovirus infection was observed in 2 cases (18\%), and the cause of death in those cases was pneumonia.

Summary of family environment in infants with otitis media was showed in Table 2. All infants were bottle-feeding babies. Each case number of them is subjected in that in Table 1 .

Case no. 2: An 8-month-old male infant.

He developed wheezing about 2 months before death, and was on medication in diagnosing with bronchitis in a doctor's office. He fed poorly and had nasal discharge without fever 3 days before death. He was found dead with his face down by his father who slept alongside him. He was pronounced dead without resuming his heartbeat during 
cardiopulmonary resuscitation in a hospital. At autopsy, otitis media with viscid serous fluid in bilateral tympanic cavity and cytomegalovirus infection in the parotid grand were observed. Additionally, hypertrophied tonsil with inflammation, tonsillitis, was observed. Lidocaine used during CPR was slightly detected in blood. As shown in Table 2, mother and father were smokers.

Case no. 7: A 4-month-old female infant (Fig.1).

She developed a cough without fever about 1 month before death. But, her mother did not bring her baby to a hospital, because she felt no concern on her baby's health. The day of death, she fed poorly in the morning in a day-care center for children, and her physical condition went suddenly wrong in the afternoon. She was transported to a hospital in the condition of cardiopulmonary arrest on arrival. She died 1 hour after admission to the hospital without resuming her heartbeat during CPR. At autopsy, otitis media with viscid and palish yellow purulent fluid in bilateral tympanic cavity and CMV infection in the parotid grand were observed. The cause of death was viral pneumonia. As shown in Table 2, parents were smokers. Drug was not detected.

Case no. 11: A 7-month-old female infant.

She was pronounced a diagnosis of pneumonia six months before death in a doctor's office, and then recovered after getting outpatient treatment in other general hospital during a short period. She developed a fever with cough and vomiting again 8 days before death, and 
the mother received 4 day's medication, antibiotics and cough suppressant for her baby in the doctor's office. The baby was found dead on her back by the mother who slept alongside her after giving her baby its bottle. The baby was pronounced dead without resuming her heartbeat during CPR in a hospital. At autopsy, otitis media with viscid serous fluid in bilateral tympanic cavity was observed, but CMV infection was not detected. However, very slight bronchitis without pneumonia, which seemed not to be cause of death, was observed, cause of death was diagnosed with SIDS. Lidocaine used during CPR and cotinine, metabolite of nicotine, was detected in blood. The concentration of cotinine was $14.6 \mathrm{ng} / \mathrm{ml}$. As father was between marriages, his smoking information could not be obtained. Mother and grandmother living together were smokers.

\section{Discussion}

Several authors [1-3] indicate that otitis media in infants is a prognostic factor for hearing performance in child and it may affect verbal development and intellectual ability. From this point of view, it seems significantly that we could detect 3 cases $(27 \%)$ with otitis media of 11 unexpected natural deaths in our study, although the number of investigated cases was few.

Engel et al. $[4,5]$ reported that both intrinsic and extrinsic factors appear to play an important role in the prevalence of otitis media with effusion, and the prevalence was most strongly associated with age. The peak prevalence was observed around the age of 10 months 
and gradually decreased with aging. Our cases were from 4 to 8 months of age.

Ey et al. [6] reported that heavy maternal smoking is a significant risk for otitis media in the first year of life, but no association was found with paternal smoking. Dybing et al. [7] also reported that the number of new episodes and duration of otitis media with effusion in young children is positively correlated with environmental tobacco smoke, and infant with lower birth weight had a high risk of recurrent otitis media during the first year of life. In our 3 autopsy cases with otitis media, all of mothers were smokers. Tobacco smoke exposure in the home may affect pathogenesis of otitis media in our cases.

Rajs et al. [8] indicated that a relationship was found between high nicotine concentration and otitis media. Tutka et al. [9] described usefulness of detecting nicotine and cotinine in connection with exposure to environmental tobacco smoke. We detected cotinine, metabolite of nicotine, in 1 case with otitis media.

Ehehardt [10] reported that otitis media alone as a cause of death is seldom, and otitis media is important, together with other respiratory disease. Of our 3 cases with otitis media, 2 cases showed pneumonia and CMV infection. It is well known that CMV infection is often observed immunocompromised patient. CMV infection and otitis media in infants seem to indicate that there is a background of hidden dysfunction or immature function in the immune system. 
Conflict of Interest: None

2

3

4

5

6 


\section{References}

[1] Creps CL, Vernon-Feagans L. Infant daycare and otitis media: multiple influences on children's later development. J Appl Dev Psychol 2000; 21: 357-378.

[2] Matubara K, Omori K, Baba K. Acute coalescent mastoiditis and acoustic sequelae in an infant with severe congenital neutropenia. Int J Pediatr Otorhinolaryngol 2002; 62: 63-67.

[3] Zielhuis GA, Gerritsen AAM, Gorissen WHM, Dekker LJ, Rovers MM, van der Wilt GJ, Ingels K. Hearing deficits at school age; the predictive value of otitis media in infants. Int J Pediatr Otorhinolaryngol 1998; 44: 227-234.

[4] Engel J, Anteunis L, Volovics A, Hendriks J, Marres E. Risk factors of otitis media with effusion during infancy. Int J Pediatr Otorhinolaryngol 1999; 48: 239-249.

[5] Engel J, Anteunis L, Volovics A, Hendriks J, Marres E. Prevalence rates of otitis media with effusion from 0 to 2 years of age: healthy-born versus high-risk-born infants. Int $\mathrm{J}$ Pediatr Otorhinolaryngol 1999; 47: 234-251.

[6] Ey JL, Holberg CJ, Aldous MB, Wright AL, Martinez FD, Taussig LM. Passive smoke exposure and otitis media in the first year of life. Pediatrics 1995; 95: 670-677.

[7] Dybing E, Sanner T. Passive smoking, sudden infant death syndrome (SIDS) and childhood infections. Hum Exp Toxicol 1999; 18: 202-205.

[8] Rajs J, Raesten-Almqvist P, Falck G, Eksborg S, Andersson BS. Sudden infant death syndrome: postmortem findings of nicotine and cotinine in pericardial fluid of infants in 
relation to morphological changes and position at death. Pediatr Pathol Lab Med 1997;

17: 83-97.

[9] Tutka P, Wielosz M, Zatonski W. Exposure to environmental tobacco smoke and children health. Int J Occup Med Environ Health 2002; 15: 325-335.

[10] Ehrhardt G. About correlation between sudden unexpected death and otitis media occulta in infancy and childhood. Zentralbl Allg Pathol 1975; 119: 91-99. 


\section{Legends to figures}

Fig. 1. Otitis media and cytomegalovirus infection observed in a 4-month-old female infant

(case no. 7 in table 1) who died of pneumonia. (A) Smear of fluid in the tympanic cavity. HE, $\times 200$ (B) Purulent fluid in the tympanic cavity opened. (C) Parotid gland. HE, $\times 400$ 
Table 1

Autopsy cases investigated in unexpected natural death of infants under one year old

\begin{tabular}{ccllll}
\hline $\begin{array}{c}\text { Case } \\
\text { no. }\end{array}$ & $\begin{array}{c}\text { Age } \\
\text { (month) }\end{array}$ & Sex & Cause of death & Otitis media & CMV infection \\
\hline 1 & 5 & Female & Pneumonia & & + \\
2 & 8 & Male & Pneumonia & + & + \\
3 & 2 & Male & Pneumonia & & + \\
4 & 8 & Male & Pneumonia & \\
5 & 4 & Male & SIDS & + \\
6 & 1 & Female & Pneumonia & \\
7 & 4 & Female & Pneumonia & + \\
8 & 4 & Female & SIDS & + \\
9 & 5 & Male & SIDS & \\
10 & 3 & Female & SIDS & \\
11 & 7 & Female & SIDS & + \\
\hline
\end{tabular}

CMV: Cytomegalovirus 
Table 2

Summary of 3 autopsy cases of infant with otitis media

\begin{tabular}{llll}
\hline & Case no. 2 & Case no. 7 & Case no. 11 \\
\hline Age & 8 months & 4 months & 7 months \\
Sex & Male & Female & Female \\
Period of death & May & October & April \\
Cause of death & Pneumonia & Pneumonia & SIDS \\
Father (Age) & Smoker (27) & Smoker (27) & Between Marriages \\
Mother (Age) & Smoker (25) & Smoker (27) & Smoker (26) \\
Number of siblings & \multicolumn{1}{c}{1} & 1 & 2 \\
Birth weight & 2688 g & $3362 \mathrm{~g}$ & $3124 \mathrm{~g}$ \\
Gestational age & 37 weeks & 38 weeks & Preterm delivery \\
Type of delivery & Vaginal & Caesarean & Caesarean \\
Pathological findings & Otitis media & Otitis media & Otitis media \\
& Tonsilitis & Pneumonia & Bronchitis \\
& Pneumonia & CMV infection & \\
& CMV infection & & \\
\hline
\end{tabular}

Case number is subjected to that in table 1 . 